\title{
Deadline-constrained Co-evolutionary Genetic Algorithm for Scientific Workflow Scheduling in Cloud Computing
}

\author{
Li Liu', Miao Zhang ${ }^{2,1, *}$, Rajkumar Buyya ${ }^{3}$, Qi Fan'
${ }^{1}$
${ }^{1}$ School of Automation and Electrical EngineeringUniversity of Science and Technology Beijing, China
${ }^{2}$ Beijing Institute of Technology, Beijing, China
${ }^{3}$ The University of Melbourne, Australia
"Corresponding Author:S20130926@ ustb.edu.cn
}

\begin{abstract}
The Cloud infrastructures provide a suitable environment for the execution of large-scale scientific workflow application. However, it raises new challenges to efficiently allocate resources for the workflow application and also to meet the user's quality of service (QoS) requirements. In this paper, we propose an adaptive penalty function for the strict constraints compared with other genetic algorithms. Moreover, the co-evolution approach is utilized to adjust the crossover and mutation probability which is able to accelerate the convergence and prevent the prematurity. We also compare our algorithm with baselines such as random, PSO, HEFT and GA in a WorkflowSim simulator on four representative scientific workflows. The results show that it performs better than other the state-of-the-art algorithms in the criterion of both the deadline-constraint meeting probability and the total execution cost.
\end{abstract}

Keywords: Cloud Computing; Scientific Workflow; Resource Scheduling; Co-evolutionary Genetic Algorithm

\section{Introduction}

Scientific experiments are usually represented as workflows [1], where tasks are linked according to their data flow and compute dependencies. Such scientific workflows are data-intensive and compute-intensive applications, for example, Compact Muon Solenoid (CMS) experiment for the Large Hadron Collider (LHC) at CERN [2] produces a huge amount of data to be analyzed, which are more than five peta-bytes per year when running at peak performance. The Human Genome Project is aimed at sequencing and identifying all three billion chemical units in the human genetic instruction set, and discovering the genetic roots of disease to find treatments. These scientific workflows have tremendous data and computing requirements, and need a high-performance computing environment for execution. Cloud computing is the latest development of distributed computing, grid computing and parallel computing [3] [4], which delivers the dynamically scaling computing resources as a utility, much like how water and electricity were delivered to households these days. The patterns that Cloud computing provides resources contain: Infrastructure-as-a-Service (IaaS), Platform-as-a-Service (PaaS), and Software-as-a-Service (SaaS) [3][5]. In this paper, we refer to IaaS Cloud which offers us a virtual pool to provide unlimited virtual machines (VMs).

This is the author manuscript accepted for publication and has undergone full peer review but has not been through the copyediting, typesetting, pagination and proofreading process, which may lead to differences between this version and the Version of Record. Please cite this article as doi: 10.1002/cpe.3942 
The main character of Cloud computing is virtualization. Cloud enables to provide computational resources in the form of virtual machines (VMs). A process that maps tasks in a workflow to compute resources (VMs) for execution (preserving dependencies between tasks) is called workflow scheduling. There are two layers for Cloud workflow scheduling which include VM-task mapping and the execution order for tasks in a single VM. In this paper, we just use the evolutionary approach to schedule VM-task mapping in the first layer, and the execution order in a single VM is set as that in paper [6], where the VM will schedule the task with the smallest end time. There are different optimization objectives for workflow scheduling in Cloud, including makespan, cost, throughput, and load balancing. In this paper, the optimization objective is the cost of executing a scientific workflow and subject to a deadline constraint, which is to find a proper task-VM mapping strategy which minimizes the total financial cost and the makespan satisfies the deadline constraint.

The context above is a constrained optimization problem, and in order to transform constrained problem into unconstrained one, most evolutionary algorithms usually use static penalty function to penalize infeasible solutions by reducing their fitness values in proportion to the degrees of constraint violation. However, it is difficult to set a suitable penalty factor. Another common method is to eliminate the infeasible individuals within their evolutionary process. However, some infeasible individuals usually hold very rarely and excellent gens to be very valuable for the next generations, and unable to be eliminated. We propose a co-evolutionary genetic algorithm with adaptive penalty function for the constrained scientific workflow scheduling in Clouds. We have considered the main features of Cloud providers such as heterogeneous computing resources and dynamic providing. An adaptive penalty function is applied in the coevolutionary genetic algorithm which will adjust itself automatically during the evolution. And we apply the notion of co-evolution to adjust the crossover and mutation probability factors which are helpful for the convergence. Our main contributions can be summarized as follows: 1) Present the optimization model of scientific workflow scheduling in cloud environment which is cost-minimization and deadline-constrained, and considers Cloud resources' dynamic provision pattern and heterogenetic character. 2) Propose a new Co-evolutionary Genetic Algorithm with Adaptive penalty function approach $C G A^{2}$, for deadline constrained scientific workflow scheduling in Clouds. It applies a self-adaptive penalty function into the coevolutionary GA which is able to prevent premature efficiently, and uses the notion of co-evolution to adjust the crossover and mutation probabilities which can efficient accelerate the convergence. 3) Unlike existing genetic approaches, we generate the initial population based on the critical path [7], which can also prevent premature efficiently and improve deadline meeting for workflow scheduling. Simulation results demonstrate that our approach have high accuracy in terms of deadline constraint satisfaction at a lower costs.

The remainder of this paper is organized as follows: the following section introduces the related works. The context of our model is presented in Section 3. Section 4presents the $C G A^{2}$ approach and the adaptive penalty function. Section 5 applies the proposed approach $\left(C G A^{2}\right)$ to Cloud scientific workflow scheduling problem. Section 6 evaluates the performance of the $C G A^{2}$, and has made comparison with the existing algorithms, then gives the experiment results. We conclude the paper with a discussion and a description of future work in Section 7.

\section{Related Work}

To address the problem of constrained workflow scheduling in Cloud Computing, some evolutionary 
algorithms have been adopted to generate near-optimal solutions. Wang and Yeo et al.[8] presented a look ahead genetic algorithm (LAGA) which utilized the reliability-driven (RD) reputation to evaluate the resource's reliability. Moreover the multi-objective model aiming to optimize both the makespan and the reliability of a workflow application was proposed. A Particle Swarm Optimization (PSO) based approach was proposed in [9], which aimed to minimizing the execution cost of a workflow while balancing the task load on the available resources. Rodriguez and Buyya [6] presented a cost-minimization and deadlineconstrained PSO approach for Cloud scientific workflow scheduling. It considered fundamental features of IaaS providers, like elastic providing and heterogeneous computing resources (VMs). A penalty function was used in their algorithms that the particles violating the constraints are inferior to the feasible particles. However this method would lead a premature convergence which is very common in the PSO algorithms.

Sawant [10] utilized the genetic algorithm (GA) for virtual machines configuration in Cloud Computing. It incorporated the constraints into the objective fitness function, so as to transform the constrained optimization problem to the unconstrained one. This is the most popular handling way for the constrained optimization problem and particularly easy to implementation. But it is very hard to set a suitable penalty factor to tradeoff between the global optimization searching and the constraints satisfying. The coefficients they used have none physical meaning and obtained through empirical evaluation. Huang [11] proposed a new improved genetic algorithm, where the chromosomes is not only representing the computing resourcetask assignment but also indicating the queue on the VMs the task being executed. It firstly evolves individuals according to the optimization objective, and changes to evolve population based on the constrained objective when the individuals violate the constraint. This approach releases the burden of devising an appropriate penalty function for constrained optimization problem. However it needs to evolve for numerous generations and a feasible solution may be unfounded.

The ant colony optimization approach was used for VMs configuration in cloud computing aiming to energy efficient[12]. The experiments showed that the proposed approach achieved superior energy gains through better server utilization and required less resource than First-Fit Decreasing approach. A particle swarm optimization (PSO) for workflow scheduling in cloud computing was proposed in [13], which considered both the computation cost and the data transmission cost. The experiments showed that the PSO can achieve as much as 3 times cost savings, and the workload is better than the existing Best Resource Selection (BRS) algorithm.

\section{Problem Formulation}

A workflow is depicted as $G=(V, E)$, where $V=\left\{t_{1}, t_{2}, \ldots, t_{n}\right\}$ and $E$ is the vertices and edges of the graph, respectively. Each vertex represents a task tand there are $\mathrm{n}$ tasks in the workflow. The edges maintain execution precedence constraints. Having a directed edge $\mathrm{e}_{\mathrm{x}, \mathrm{y}}$ from $t_{x}$ to $t_{y}, \mathrm{x}, \mathrm{y} \in \mathrm{M}$ means that $t_{y}$ can't start to execute until $t_{x}$ is completed, task $t_{x}$ is a parent task of $t_{y}, t_{y}$ is a child task of $t_{x}$. Tasks without parents are called the entry task $t_{\text {entry }}$, and tasks without child tasks are called the exit task $t_{\text {exit }}$. Each workflow has a deadline $d_{W}$ associated to what determines the allowed longest time to complete its execution. Fig. 1 shows an example of workflow, in which each node represents a task and the arcs show the data transfer between nodes. 
The IaaS Cloud providers offer a range of VM types denoted by $\overline{V M}=V M_{1}, V M_{2}, \ldots, V M_{n}$. Different VM types provide different computing resources, and then we define VM type in terms of its processing capacity $P_{V M_{i}}$ and cost per unit of time $C_{V M_{i}}$. VMs are charged per unit of time $\tau$, if $\tau=60$ minutes, a utilization of VM for 61 minutes would incur a payment of two hours (two units of time). We assume each task is executed by a single VM, and a VM can execute several tasks.

The running time $R T_{t_{i}}^{V M_{t_{i}}}$ of task $t_{\mathrm{i}}$ executed by $V M_{t_{i}}$ is calculated in Eq.1, where $s_{t_{i}}$ is the size of taskt $t_{i}, P_{V M_{t_{i}}}$ is the processing capacity of $V M_{t_{i}}$. The transfer time $T T_{e_{i, j}}$ between a parent task $t_{i}$ and its child task $t_{j}$ is depicted in Eq.2, where $\mathrm{d}_{\mathrm{t}_{\mathrm{i}}}^{\text {out }}$ is the output data size produced by task $t_{i}, \beta$ is the bandwidth between each VM, and the bandwidth for all VMs are roughly same. If two tasks are executed in the same VM, the transfer time is 0 .

$$
\begin{aligned}
& R T_{t_{i}}^{V M_{t_{i}}}=s_{t_{i}} / P_{V M_{t_{i}}} \\
& T T_{e_{i, j}}=d_{t_{i}}^{\text {out }} / \beta
\end{aligned}
$$

There are many different optimization objectives for workflow scheduling in Clouds. In this paper, we focus on finding the optimization solution for workflow scheduling which can minimize the total execution cost and satisfy the deadline constraint. We define a scheduling vector $S=(M, T E C, T E T)$ in terms of tasks to resources matching $\mathrm{M}$, the total execution cost TEC and the total execution time TET. $\mathrm{M}$ is the task-VMs matching which is comprised of $\mathrm{VM}$ types, start time and end time for all tasks, $M=\left(m_{t_{1}}^{V M_{t_{1}}}, m_{t_{2}}^{V M_{t_{2}}}, \ldots, m_{t_{M}}^{V M_{t_{M}}}\right), m_{t_{i}}^{V M_{t_{i}}}=\left(t_{i}, V M_{t_{i}}, S T_{t_{i}}, E T_{t_{i}}\right)$, which means task $t_{\mathrm{i}}$ is associated with $\mathrm{VM}_{\mathrm{t}_{\mathrm{i}}}$, and the start time and the end time for task $\mathrm{t}_{\mathrm{i}}$ is calculated by Eq.3and Eq.4.

$$
\begin{aligned}
& S T_{t_{i}}=\left\{\begin{array}{cc}
\operatorname{LET}_{V M_{t_{i}}}, & \text { if } t_{i} \text { is a entry task } \\
\max \left(\begin{array}{c}
\max \\
t_{a} \in{\operatorname{parent}\left(t_{i}\right)}
\end{array}\left(E T_{t_{a}}+T T_{e_{a, i}}\right), L E T_{V M_{t_{i}}}\right) \text { otherwise }
\end{array}\right. \\
& E T_{t_{i}}=S T_{t_{i}}+R T_{t_{i}}^{V M_{t_{i}}}
\end{aligned}
$$

where $L E T_{V M_{t_{i}}}$ is the lease end time of $V M_{t_{i}}$, which is also the time that $V M_{t_{i}}$ becomes idle.There will be no charge for data transfers within a same data center, and we do not consider this fee when calculating the workflow total cost. The total execution cost TEC and the total execution time TET are calculated as Eq.5and Eq.6 respectively.

$$
T E C=\sum_{i=1}^{|V M|} C_{V M_{t_{i}}} *\left[\frac{R T_{t_{i}}^{V M_{t_{i}}}}{\tau}\right]+\sum_{i \in T, j \in T} T T_{e_{i, j}} * T C_{V M_{t_{i}}}
$$




$$
T E T=\max \left(E T_{t_{i}}: t_{i} \in V\right)
$$

where $C_{V M_{t_{i}}}$ is the processing cost for $V M_{t_{i}}$, and $T C_{V M_{t_{i}}}$ is the data transfer cost for $V M_{t_{i}}$.

The problem in this paper can be described as finding a scheme $S$ with minimum the TEC, and the TET do not exceed the workflow's deadline constraint $d_{W}$. As showed in Eq.7.

Minimize TEC

Subject to: $T E T \leq d_{W}$

In this paper, we use a Co-evolutionary Genetic Algorithm with Adaptive penalty function approach $\left(C G A^{2}\right)$ to solve this optimizing problem. Table 1 gives description of notations used in our paper.

\section{Co-evolutionary GA with Adaptive penalty function}

In this section, we have described a Co-evolutionary Genetic Algorithm with Adaptive penalty function approach $\left(C G A^{2}\right)$ to solve constrained optimization problem.

\subsection{Mechanism of the Co-evolutionary GA}

Co-evolution is the process of mutual adaptation of two or more populations. The key issue in the coevolutionary algorithms is that the evolution of a population depends on another population.

In 1994, Paredis[15] introduced Co-evolutionary Genetic Algorithm (CGA). Coello[16] incorporated the notion of co-evolution into a GA to adapt genetic coefficients and for solving constrained optimization problems. In this work, we will employ the notion of co-evolution to adjust the crossover and mutation probabilities, and apply an adaptive penalty function coefficients scheme in the genetic algorithm.

The structure of co-evolution model in $\mathrm{CGA}^{2}$ is shown in Fig. 2. In $\mathrm{CGA}^{2}$, two types of populations are used. In particular, one type of a single population (denoted by Population ${ }_{2}$ ) with size $\mathrm{M}_{2}$ is used to adapt suitable crossover and mutation probability factors, and another kind of multiple populations (denoted by Population $_{1,1}$, Population $_{1,2}, \ldots$, Population $_{1, M_{2}}$ ) that each of them is with size $\mathrm{M}_{1}$ evolves in parallel with different crossover and mutation schemes to search good decision solutions. Each individual $B_{j}$ in Population $_{2}$ represents a set of crossover and mutation probability factors for individuals inPopulation Poj $_{1, j}$, where each individual represents a decision solution.

In every generation of co-evolution process, each Population $_{1, j}$ will evolve by the GA for a certain number of generations $\left(G_{1}\right)$ with crossover and mutation probability factors obtained from individual $\mathrm{B}_{\mathrm{j}}$ in Population P $_{2}$ Then the fitness of each individual $\mathrm{B}_{\mathrm{j}}$ in Population $\mathrm{P}_{2}$ will be determined. After all indi- 


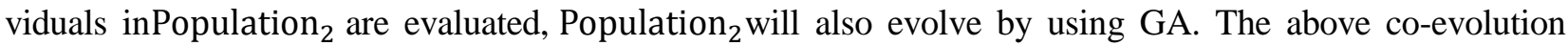
process will repeat until a pre-defined stopping criterion is satisfied (e.g., a maximum number of co-evolution generation $G_{2}$ is reached).

In short, two types of populations evolve interactively, where Population $_{1, j}$ with an adaptive penalty function scheme is used to evolve decision solutions, while Population ${ }_{2}$ is used to adapt crossover and mutation probabilities for solution evaluation. Due to the co-evolution, not only decision solutions are explored evolutionary, but also crossover and mutation probabilities are adjusted in a self-tuning way to avoid the difficulty of setting suitable factors by trial and error[14].

\subsection{Adaptive crossover and mutation probabilities}

In genetic algorithm, the bigger the crossover probabilityp $\mathrm{x}_{\mathrm{x}}$ and mutation probability $\mathrm{p}_{\mathrm{m}}$ are, the more new individuals will be generated along with the diversity of population. But if the probabilities are too big, good genes will be destroyed easily, otherwise if the probabilities are too small, it is not conducive to generate new individuals and the search speed will slow down [17].

According to Zhang et al. [18], the evolutionary process in GA can be depicted as four states, including initial state, sub-maturing state, maturing state and matured state. The crossover probability $\mathrm{p}_{\mathrm{x}}$ and mutation probability $\mathrm{p}_{\mathrm{m}}$ are adjusted differently based on population states. For example, if there are almost inferior individuals with extremely poor fitness, we should increase $p_{m}$ and decrease $p_{x}$, like in initial state. If the span of fitness values in a population is very large, the crossover probability $p_{x}$ should be increased, like sub-maturing state and maturing state. If there are almost excellent individual with good fitness, we could decrease $\mathrm{p}_{\mathrm{x}}$ and $\mathrm{p}_{\mathrm{m}}$ as in the matured state. According these rules and inspired by the idea in the literature [27] [28], a self-adaptive crossover and mutation operator is described as Eq.8 and Eq.9.

$$
\begin{gathered}
p_{x}(i)=\omega_{1} * \cos \left(\frac{\pi}{2} * \frac{1}{e^{\left(\sigma_{1}(i)+\sigma_{2}(i)+\cdots+\sigma_{m}(i)\right)}}\right) \\
p_{m}(i)=\omega_{2} * f_{i} / f_{p}
\end{gathered}
$$

where $p_{x}(i)$ is the crossover probability for $\mathrm{i}$-th individual and $\mathrm{p}_{\mathrm{m}}(\mathrm{i})$ is the mutation probability. $\sigma_{m}(i)=$ $\left|f_{i}-f_{i}(m)\right| / f_{i}, f_{i}(m)$ is the $m$-th closest individual to individual $\mathrm{i}$ in terms of fitness value. $f_{i}$ is the fitness value of individual $i$, and $f_{p}$ is the individual with biggest fitness value in the population. $\omega_{1}$ and $\omega_{2}$ are the crossover and mutationprobability factors which are adapted through co-evolution.

\subsection{Fitness calculation with Adaptive penalty function}

A suitable penalty function plays an important role in the performance of GAs, and it becomes more important when the constraints are stricter which means that the optimal solution is nearing to the boundary between the feasible and infeasible search space [19][29]. The most common form of the penalized fitness function is depicted as following:

$$
F_{i}^{a}=F^{a}\left(x_{i}\right)=F\left(x_{i}\right)-P\left(x_{i}\right)=F\left(x_{i}\right)+\sum_{j=1}^{m} \lambda(j) * E_{j}\left(x_{i}\right)
$$


where $F_{i}^{a}$ is the fitness function of $\mathrm{i}$-th individual after penalty. $F\left(x_{i}\right)$ is the fitness value for the optimizing objective, $\lambda(j)$ is the penalty factor for the $\mathrm{j}$-th constraints violation, $\mathrm{m}$ is the number of constraints, and $E_{j}\left(x_{i}\right)$ is the $\mathrm{j}$-th constraint violation for $\mathrm{i}$-th individual.

Inspired by Nanakorn et al. [20], the penalty function should be suitable for all infeasible individuals. If it is too small, many infeasible individuals may have higher penalized fitness value, and the population would move towards a false direction to the infeasible region. Otherwise, if it is too large, some individuals with good gens will be eliminated which may lead to premature convergence. According to Tessema et al. [21], an adaptive penalty function strategy is applied to keep track of the number of feasible individuals in the population to determine the amount of penalty added to the infeasible individuals.

Firstly, each individual's fitness value and constraint violation will be normalized by the Eq.11 and Eq.12.

$$
\tilde{F}\left(x_{i}\right)=\frac{F\left(x_{i}\right)-F_{\min }}{F_{\max }-F_{\min }}
$$

where $\tilde{F}\left(x_{i}\right)$ is the normalized fitness value, $F_{\min }$ and $F_{\max }$ are the smallest and the largest fitness value for all individuals in the current population. In this way, each individual's fitness will lie between 0 and 1 . And the normalized constraint violation $\tilde{E}\left(x_{i}\right)$ of each infeasible individual is calculated as:

$$
\tilde{E}\left(x_{i}\right)=\frac{1}{m} \sum_{j=1}^{m} \frac{E_{j}\left(x_{i}\right)}{E_{j}^{\text {max }}}
$$

where $m$ is the number of constraints, $E_{j}^{m a x}$ is the maximum violation for $j$-th constraints for all infeasible individual.

Furthermore, for different evolutionary states in GA, the penalty rule should be adapted accordingly. For example, if there are few feasible individuals in the population, the infeasible individuals with lower constraint violation will be less penalized than those with higher constraint violation. On the other hand, if there are many feasible individuals in the population, the infeasible individual with lower normalized fitness should be less penalized. According to literature [19] [21], the final fitness value is formulated in the follow.

(1) If there is at least one feasible individual in the current population, the fitness function is as Eq.13

$$
F 1^{a}\left(x_{i}\right)= \begin{cases}\tilde{F}\left(x_{i}\right) & \text { for feasible individual } \\ \sqrt{\tilde{F}\left(x_{i}\right)^{2}+\tilde{E}\left(x_{i}\right)^{2}}+\left[\left(1-r_{f}\right) \tilde{E}\left(x_{i}\right)+r_{f} \tilde{F}\left(x_{i}\right)\right] & \text { otherwise }\end{cases}
$$

where $r_{f}=\frac{\text { number of feasible individuals }}{\text { population size }}$. In this way, the individuals with both low fitness value and low constraint violation will be considered better than those with high fitness value or high constraint violation. And if the feasibility ratio $\left(r_{f}\right)$ in the population is small, then the individual that is closer to the feasible space will be considered better. Otherwise, the individual with lower normalized fitness value will be better. 
Eq.14.

(2) If there is no feasible individual in the current population, the fitness function is calculated as

$$
F 1^{a}\left(x_{i}\right)=\tilde{E}\left(x_{i}\right)
$$

Obviously, the individuals with smaller constraints violation are considered better. Consequently, the search will move to the region where the sum of constraints violation is small (i.e. the boundary of the feasible region).

The fitness value for i-th individual in Population $_{1, j}$ in $C G A^{2}$ is evaluated based on Eq.13 and Eq. 14.

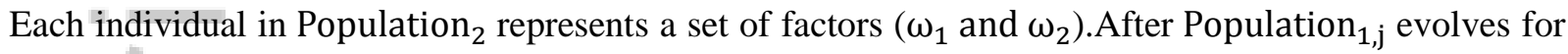
$G_{1}$ generations, the jth individual $B_{j}$ in Population ${ }_{2}$ is evaluated by Eq. 15 .

$$
\mathrm{F} 2\left(\mathrm{~B}_{\mathrm{j}}\right)=-\min \left(\mathrm{F} 1_{\mathrm{j}}\right)+\frac{\text { numinfeasible }}{\mathrm{M}_{1}}
$$

where $F 1_{j}$ is the fitness values for all individuals in Population $_{1, j}$, numinfeasible is the number of infeasible individuals in Population $_{1, j}, M_{1}$ is the size of Population Po $_{1, j}$.

\section{TheCGA ${ }^{2}$ for Workflow Scheduling}

\subsection{CGA $^{2}$ Modeling}

In this paper, we use two types of chromosomes to model $C G A^{2}$ for scientific workflow scheduling in Clouds. As shown in Fig.3, the chromesome $_{i, j}$ in Population $_{1, j}$ represents the decision solution which is also the ordered pair of task-resource matching of a workflow. For the scheduling scenario here, the position of each gene in chromosome $_{i, j}$ is the task number, and the value of each gene in chromosome $_{i, j}$ is the VMs' number, thus the dimension of a chromosome $i, j$ is the number of tasks in a workflow. The range of genes in chromosome $_{i, j}$ is determined by the number of resource available to run the tasks. Fig.3 represents a workflow with 8 tasks and 5 VMs available. The fitness function is used to determine how good a decision solution is, which is calculated by the optimizing objective total execution cost $T E C$ and the constraint total execution time TET. The calculation of TEC and TET for a chromosome are explained in next section.

The chromosome $_{m}$ in Population $_{2}$ represent the crossover and mutation probability coefficients, which is defined by the binary encoding. The range of $\omega_{1}$ is $(0,1]$, and we use the first 7 genes to represent 
the coefficient $\omega_{1}$. The value of $\omega_{1}$ in chromosome $_{m}$ is calculated as $\omega_{1}=\frac{2^{6}+2^{4}+2^{2}+1}{128}=0.6640625$. The range of $\omega_{2}$ is also $(0,1]$, and we use the latter 7 genes to represent the coefficient $\omega_{2}$ And the value of $\omega_{2}$ is calculated as $\omega_{2}=\frac{2^{5}+2^{3}+1}{128}=0.3203125$.

The Population P $_{1, j}$ is the evolution decision solutions to match the task with resource to minimize the

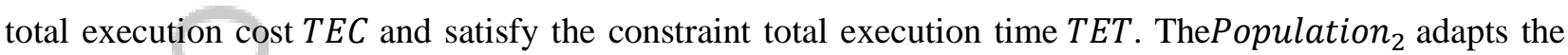
crossover and mutation probabilities for solution evolution.

\subsection{TEC and TET Calculation}

To address the workflow scheduling problem, we need to estimate the running time of workflow application with a specific task-VM mapping schedule firstly and calculate the cost accordingly. The total execution cost TEC and the total execution time TET of a chromosome $_{i, j}$ in Population $_{1, j}$ is shown in Algorithm 1. The $\mathrm{k}$-th position value of chromosome $_{i, j}(k)$ represents that task $k$ is associated withV $M_{\text {chromosome }_{i, j}(k)}$. InPopulation,$j$, a chromosome is a task-resource match.

Firstly, we initial the VMs state matrix VS and the task state matrixTS.Aset of workflow tasks T and a set of VMs $\overline{V M}$ are inputted. Then we estimate the execution time $R T_{t_{i}}^{V M_{t_{i}}}$ of each workflow task $t_{i}\left(t_{i} \in T\right)$ on every type of $\operatorname{VM} V M_{i}\left(V M_{i} \in \overline{V M}\right)$ according Eq.1, and transfer time $T T_{e_{i, j}}$ between tasks is calculated according Eq. 2.

The starting time value $S T_{t_{i}}$ has two cases. If the task has no parent tasks, it can start as soon as the VM assigned to the task is idle. Otherwise, the task starts after the parent tasks finished and the output data transferred. Furthermore, if the VM is still busy, the starting time has to be delayed until the VM enable. And in our algorithm, if two tasks allocated on the same VM have the same start time, the VM will process the task with smaller size. The ending time value $\mathrm{ET}_{\mathrm{t}_{\mathrm{i}}}$ is calculated by Eq.4 based on the starting time and execution time $R T_{t_{i}}^{V M_{t_{i}}}$. After a task has been scheduled, we need to update the VS and the TS to set the task $t_{i}$ as scheduled and the time period between $S T_{t_{i}}$ and $E T_{t_{i}}$ as busy for $V M_{t_{i}}$. The process continues until all tasks having been scheduled.

\section{ALGORITHM 1 TEC AND TET ESTIMATION}

Input: a set of workflow tasks $T$, a set of $V M s \overline{V M}$, and a chromosome ${ }_{k, j}$ in populationion $_{1, j}$ Output: TEC and TET 
1. Initial VMs state matrix VS and task state matrix TS.

2.Calculate execution time RT $[|T| \times|\overline{V M}|]$;

Calculate transfer time TT $[|T| \times|T|]$;

3. For $\mathbf{i}=1:|T|$

If $\operatorname{TS}(T(i))$ is unscheduled

3.1. $t_{i}=T(i), V M_{t_{i}}=v m_{\text {chromosome }_{k, j}(i)}$

3.2. If $t_{i}$ has no parents

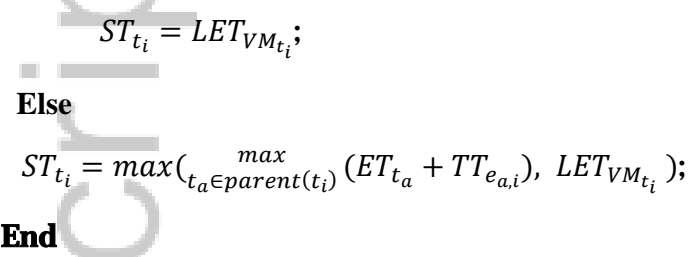

3.3. For each child task $t_{c}$ of $t_{i}$ If $t_{c}$ is mapped to a $V M$ different to $V M_{t_{i}}$ $T T(i)=T T(i)+T T(i, c)$

\section{End}

\section{End}

3.4. $R T_{t_{i}}^{V M_{t_{i}}}=R T\left(t_{i}, V M_{t_{i}}\right)$;

3.5.ET $T_{t_{i}}=R T_{t_{i}}^{V M_{t_{i}}}+T T(i)$

3.6.update VS, and TS, set the time period $\left[S T_{t_{i}}, E T_{t_{i}}\right]$ for $V M_{t_{i}}$ is busy, set TS $(T(i))$ as scheduled.

End

End

4.Calculate TEC according Eq.5;

5. Calculate TET according Eq.6;

\subsection{Initial population}

For a scientific workflow, the execution time of the tasks in the Critical Path makes more influence on the total execution time of a workflow, while the financial execution cost of these tasks is a small part of the total execution cost. So allocating these tasks to the high performance VMs will decrease the total execution time greatly, while just have a little impact on the total financial execution cost.

The diversity of initial population impacts the performance of GA greatly, but most of GAs generate the initial population randomly. In order to improve the solution quality and convergence speed, we generate one fifth of the initial population based on Critical Path (CP) [7] and assign these tasks in CP to the VMs with high processing capacity. The tasks in other one fifth of the initial population are allocated to the VMs which have the lowest price. The rest of the population is produced in random.

Fig.4 shows the framework of $C G A^{2}$. We firstly initialize two types of populations, where 
Population $_{2}$ is used to adapt crossover and mutation probabilities for Population 1 to find decision solutions. In this paper, the evolution of Population $_{2}$ is an unconstrained optimizing problem which do need penalty function, while Population $_{1}$ uses an adaptive penalty function to transform the constrained Workflow scheduling problem as an unconstrained optimizing one. The initial population scheme used in Population $_{1}$ is depicted in Section 5.3, and the Population 2 adopts the random method. Each subpopulation Population $_{1, j}$ in the Population $_{1}$ will evolve for $G_{1}$ iterations simultaneously, and the best $M_{2}$ individuals from the $M_{2}$ sub-populations will be used to assess the corresponding individual in the Population $_{2}$. The Population 2 will evolve for $G_{2}$ iterations to find the best crossover and mutation probability factor and the best decision making solution.

\section{Performance Evaluation}

To evaluate the performance of $C G A^{2}$ in addressing the problem of scientific workflow scheduling in Clouds, we use the WorkflowSim framework supported by CloudSim to simulate a cloud environment. The simulated workflows are four famous scientific workflows: Epigenomics, Montage, Inspiral and Cybershake[22][23], which are widely applied for performance measurement of scheduling algorithms in the WorkflowSim [30]. Each of these workflows has different structures as seen in Fig.5 [10].

We use related approaches for constrained optimization problem, such as the Random, HEFT [24],the genetic algorithm[10] and the PSO for deadline-constrained Cloud scientific workflow scheduling [9], as a baseline to evaluate our approach.

The Random is an algorithm that assigns the ready tasks to an idle VM randomly. The Heterogeneous Earliest Finish Time (HEFT) is a scheduling algorithm that gives higher priority to the workflow task which has higher rank value. This rank value is calculated by utilizing average execution time for each task and average communication time between resources of two successive tasks, where the tasks in the CP have comparatively higher rank values. Then, it sorts the tasks by the decreasing order of their rank values, and the task with a higher rank value is given higher priority. In the resource selection phase, tasks are scheduled in the order of their priorities, and each task is assigned to the resource that can complete the task at the earliest time. We set $\left|T_{x}\right|$ as the size of task $T_{x}$ and $\mathrm{R}$ as the set of resources (VMs) available with average processing power $|R|=\sum_{i=1}^{n}\left|R_{i}\right| / n$, and the average execution time of the task is defined as $E\left(T_{x}\right)=\frac{\left|T_{x}\right|}{|R|}$

Let $\mathrm{T}_{\mathrm{xy}}$ as the size of data to be transferred between task $T_{x}$ and $T_{y}$, and $\beta$ be the bandwidth between each VM. Thus, the average data transfer time for the task is defined as $T T_{x, y}=T_{x y} / \beta . E\left(T_{x}\right)$ and $T T_{x, y}$ are used to calculate the rank of a task. Rank value is calculated as:

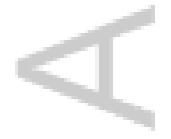




$$
\operatorname{rank}\left(T_{x}\right)=\left\{\begin{array}{cc}
E\left(T_{x}\right) T_{x} & \text { is an exit task } \\
E\left(T_{x}\right)+\underset{T_{y} \in \operatorname{child}\left(T_{x}\right)}{ }\left(T T_{x, y}+\operatorname{rank}\left(T_{y}\right)\right) \text { otherwise }
\end{array}\right.
$$

A workflow is represented as a DAG, and the rank values of the tasks in HEFT are calculated by traversing the task graph in a breadth-first search (BFS) manner in the reverse direction of task dependencies (i.e., starting from the exit tasks). The HEFT algorithm generates schedules based on VMs and tasks and does not vary with constraints.

In our experiments, we model an IaaS provider offering a single data center and five types of VMs. The VM configurations are based on current Amazon EC2 offerings and are presented in Table 2. We set processing capacity of each type of VMs based on the work of Ostermann et al. [25].

The experiments are conducted by using 4 different deadlines. These deadlines lie between the slowest and the fastest runtimes. The slowest runtime is obtained by using a single VM with the average processing capacity of all VMs to execute all tasks. And the fastest runtimes is obtained by assigning the highest processing capacity VM to the ready tasks. To estimate each of the four deadlines, the difference between the fastest and the slowest times is divided by 10 to get an interval size. The first deadline is the slowest runtime minus 1 interval sizes to the fastest deadline, as to the second one, we minus 4 interval sizes. The third is the fastest runtime adding 2 interval sizes to the slowest deadline and the last one is the fastest runtime adding 1 interval sizes.

For the testing, the parameters of $\mathrm{CGA}^{2}$ are set as follows: $M_{1}=200, G_{1}=100, M_{2}=50, G_{2}=20$. To compare the results, we consider the average workflow total execution cost and total execution time after running each experiment for 30 times. All the experiments are performed on computers with Inter Core i54570S CPU (2.9GHz and $8 \mathrm{G} \mathrm{RAM})$.

\subsection{Deadline Constraint Evaluation}

In this section, we analyze the algorithms in terms of meeting the user's defined deadlines. We have compared the deadline meeting percentages for each scientific workflow under different deadline shown as the Fig.6.

For the Epigenomics workflow, HEFT meets all of the deadlines. Random algorithm meets Deadline 1 and 2 with 10 percent and 3.3 percent respectively, and completely fails to meets Deadline 3 and 4. GA and PSO meet Deadline 1 and Deadline 2 with 100 percent, but when the constraints become strict, the rates are less and less. For Deadline 3, the constraint meeting rates for the two evolutionary algorithms are 93 percent and 73.3 percent respectively, and for Deadline 4, the rats are $26.7 \%$ and $13.3 \%$. As to our proposed CGA ${ }^{2}$ algorithm, when the constraints become stricter, $C G A^{2}$ algorithm can still find excellent solutions in terms of constraints meeting. The deadline meeting rates for first 3 deadlines are 100\%, and $80 \%$ for the last deadline constraint. The results for Montage application again show that HEFT meets all of the deadlines and it is much better than that of other algorithms. In Montage, Random algorithm obtains similar results to those obtained in Epigenomics, it meets all deadline constraints in the lowest rates. And the GA and PSO based algorithms perform well just when the deadline is relaxed like in the Deadline 1 and

Deadline 2. $C G A^{2}$ can still find excellent solutions in terms of constraints meeting when the constraints be- 
comes stricter. The meeting rates in different constraints are 100\%, 100\%, 100\%, and $60 \%$.

The results of meeting rate for Inspiral and Cybershake are much like that of the above two workflows. The Random algorithm could hardly get feasible solutions under all constraints while the HEFT gets $100 \%$ meeting rates under all constraints. As to the 3 evolutionary approaches, they perform similarly under the first two relaxed constraints, while our proposed algorithm obviously performs much better than the other two evolutionary algorithms. A possible explanation for these results revolves the fact that: HEFT always assigns tasks with VMs that make the end time at a minimum level, and it considers the entire workflow rather than focusing on only unmapped independent tasks at each step to assign priorities to tasks. But it gives no concern to the cost and constraints. Random algorithm assigns tasks with VMs randomly which is very hard to satisfy user's constraints. As to two evolutionary algorithms GA and PSO, they handle the constraints optimizing problem simply which just give a static penalty function or even exclude the solutions which violate the constraints in the evolutionary process, and it would lead to a premature convergence or a false direction to the infeasible region.

\subsection{TET Evaluation}

As shown in Fig.7, we measured the average of total execution time TET (also called as makespan) for each workflow under different constraints (red line is the TET constraint). We can see that, in the Epigenomics workflow, the HEFT approach has the lowest average makspan under every deadline constraints, and the Random has the worst performance. The GA and the PSO have a good performance in the relaxed constraints like Deadline 1 and Deadline 2. And when the constraints become stricter, they perform poorly. As to our proposed $C G A^{2}$, it shows a better performance than both the GA and the PSO, especially in the strict constraints. In the Montage workflow case, the HEFT still has the best average makespan value while the Random is the worst in terms of total execution time. In the Deadline 1 and Deadline 2, the mean of total execution time(TET) for the GA, PSO and $C G A^{2}$ are less than deadlines, while the average TET for these algorithms in the last constraints are above the deadline values.

In the Inspiral and Cybershake workflows, our proposed algorithmCGA ${ }^{2}$ still has better TET results comparing with the other two evolutionary algorithms. These results are in line with those analyzed in the deadline constraint evaluation section, from which we were able to conclude that, HEFT algorithm can obtain a lowest total execution time value for workflows, and Random algorithm is not efficient in meeting the deadlines, and the normal GA and PSO can't find excellent solutions when the constraints become stricter. On the contrary, $C G A^{2}$ exhibits a larger makespan variation which is expected as it can find excellent solution for constrained optimizing problem in a very large and chaotic search space.

\subsection{TEC Evaluation}

The average total execution costs generated from the above algorithms for each of the workflows are displayed in Fig. 8. We also give the mean of TET and deadlines meeting rates obtained in the former section, as the algorithms should be able to generate a cost-efficient scheme but not at the expense of a long 
execution time. There is unavailable for an algorithm to run on the lower cost without meeting the deadlines. The mean of TEC, the mean of TET, and the meeting rate for each workflow under different constraints are presented in Table 3.

For the Epigenomics workflow, HEFT total execution time is still the lowest one for the 4 different constraints, but its total execution cost is higher than evolutionary algorithms. In these experiments, $C G A^{2}$ get the lowest cost under each deadline which shows the optimizing capacity of our proposed algorithm is better than other previous evolutionary algorithms especially under the strict deadlines. From the table we can also find that when the constraints become stricter, the solutions obtained by the GA and PSO are not only fail to meet deadlines but also get much more cost. It shows that an inferior penalty function would lead the populations to premature and infeasible sections.

In the Montage workflow, HEFT total execution time is still the lowest for the 4 different constraints, but its total execution cost is higher than evolutionary algorithms. A possible explanation for this might be that evolutionary algorithms lease VMs with lower price in order to minimize the total execution cost. What's more, the tasks are relatively small in Montage workflow, which means that the machines in the HEFT' scheme are only running for a small amount of time but charged for the full billing period, and choosing a higher processing capacity VM means much more cost.

Among the above algorithms complying with the deadline constraint, GA and PSO can obtain the low cost schedules on relaxed deadline, but when the constraints become strict, the solutions generated by them are very poor, while the $C G A^{2}$ can still get excellent solutions in terms of the TEC meeting deadlines under strict constraints. From the results, it is clear that the evolutionary algorithms based approaches perform better than HEFT in terms of cost. And the $C G A^{2}$ can get excellent solution without violating the constraints when the deadline becomes strict.

TEC results of 5 algorithms in the Inspiral and Cybershake Workflow are similar to those of the previous workflows, which again show that $C G A^{2}$ could always find the best solutions in terms of TEC, especially under the strict constraints. We can observe from Fig.8 (c) (d) that, the GA and PSO with static penalty function have a rapid increase in terms of TEC when the constraints get stricter, while our algorithm is able to maintain the similar level. What's more, even though the PSO with static penalty function could get a better TEC results than $C G A^{2}$ in the relaxed constraints, it still could not get satisfied solutions in the strict constraints.

From the above discussion, the following conclusions can be drawn from the experiments: the Random algorithm fails to meet deadlines in most cases whereas HEFT could get the lowest makespan which always assign highest processing capacity VMs to ready tasks. While comparing with HEFT, the evolutionary algorithms are capable of generating cheaper schedules and hence outperform HEFT in terms of cost optimization. And compared to GA and PSO with static penalty functions, our proposed algorithm CGA ${ }^{2}$ can handle the constrained optimization problem of scientific workflow scheduling in Clouds better, and is still able to find feasible solutions under strict user's deadline requirements.

\section{Conclusion and Future Work}


In this paper, a co-evolutionary genetic algorithm with adaptive penalty function $\left(C G A^{2}\right)$ for constrained scientific workflow scheduling in Clouds is proposed.The common drawback of existing evolutionary algorithms is the necessity of defining problem-specific parameters of penalty function for constrained optimization problem. And these algorithms are also static and lead to premature convergence. In order to address these problems, our proposed algorithm designs an adaptive penalty function without any parameter tuning and easy to implement.

This approach could effectively exploit the information hidden in infeasible individuals which sets the proper penalty rule for the individuals at different stages of the evolutionary process. In addition, our proposed algorithm $C G A^{2}$ applies an adaptive crossover and mutation probabilities based on the co-evolution and generates the initial population according to critical path which are efficient for preventing premature and improving deadline meeting for workflow scheduling. Experiments demonstrate that, our solution has an overall better performance than the state-of-the-art algorithms Random, HEFT, GA and PSO.The $C G A^{2}$ succeeds with high rate as HEFT which aims to minimizing the makespan without considering the cost. Furthermore, $C G A^{2}$ could produce schedules with lower total executing cost and meeting the deadlines under strict constraints while GA and PSO could not succeed easily.

Our future work will use multi-objective evolutionary algorithm to solve the cloud resource scheduling problem, and will take into account the load balance and task failures. Meanwhile, we will extend the resource model to consider the data transfer cost between data centers.

\section{Acknowledgment}

This work is supported by the National Natural Science Foundation of China (Grant No. 61370132 No.61472033, No.61272432), Beijing Natural Science Foundation (No.4152034).

\section{References}

[1] Vöckler JS., Juve G., Deelman, E., Rynge M., Berriman B. Experiences using cloud computing for a scientific workflow application. In Proceedings of the 2nd international workshop on Scientific cloud computing2011;(pp. 15-24). ACM.DOI:10.1145/1996109.1996114.

[2] http://hc.web.cern.ch/lhc/LHC Experiments.htm

[3] Buyya R., Yeo CS., Venugopal S., Broberg, J, Brandic I. Cloud computing and emerging IT platforms: Vision, hype, and reality for delivering computing as the 5th utility. Future Generation computer systems2009;25(6), 599616.DOI:10.1016/j.future.2008.12.001.

[4] Foster I, Zhao Y, Raicu I, Lu S. Cloud computing and grid computing. 360-degree compared. In Grid Computing Environments Workshop, 2008. GCE'08 (pp. 1-10). IEEE.DOI: 10.1109/GCE.2008.4738445. 
[5] Liu L, Zhang M, Lin Y, Qin L. A survey on workflow management and heduling in cloud computing. In Cluster, Cloud and Grid Computing (CCGrid), 2014 14th IEEE/ACM International Symposium on (pp. 837-846). IEEE.DOI:10.1109/CCGrid.2014.83.

[6] Rodriguez MA, Buyya R. Deadline based resource provisioningand scheduling algorithm for scientific workflows on clouds. Cloud Computing, IEEE Transactions on2014;2(2), 222-235.DOI:10.1109/TCC.2014.2314655.

[7] Ma T, Buyya R. Critical-path and priority based algorithms for scheduling workflows with parameter sweep tasks on global grids. In Computer Architecture and High Performance Computing, 2005.SBAC-PAD 2005. 17th International Symposium on (pp. 251-258). IEEE.DOI:10.1109/CAHPC.2005.22.

[8] Wang X., YeoCS, Buyya R, Su J. Optimizing the makespan and reliability for workflow applications with reputation and a look-ahead genetic algorithm. Future Generation Computer2011; Systems, 27(8), 11241134.DOI:10.1016/j.future.2011.03.008.

[9] Pandey S, Wu L, Guru SM, Buyya R. A particle swarm optimization-based heuristic for scheduling workflow applications in cloud computing environments. In Advanced information networking and applications (AINA), 2010 24th IEEE international conference on (pp. 400-407). IEEE.DOI:10.1109/AINA.2010.31.

[10] Sawant S. A genetic algorithm scheduling approach for virtual machine resources in a cloud computing environment. 2011.

[11] Huang J. The workflow task scheduling algorithm based on the GA model in the cloud computing environment. Journal of Software2014;9(4), 873-880.

[12] Feller E, Rilling L, Morin C. Energy-aware ant colony based workload placement in clouds. In Proceedings of the 2011 IEEE/ACM 12th International Conference on Grid Computing (pp. 26-33).IEEE Computer Society.DOI:10.1109/Grid.2011.13.

[13] Wu Z, Ni Z, Gu L, Liu X. A revised discrete particle swarm optimization for cloud workflow scheduling. In Computational Intelligence and Security (CIS), 2010 International Conference on (pp. 184-188). IEEE. DOI:10.1109/CIS.2010.46.

[14] He Q, Wang L. An effective co-evolutionary particle swarm optimization for constrained engineering design problems. Engineering Applications of Artificial Intelligence2007;20(1), 89-99.

DOI:10.1016/j.engappai.2006.03.003.

[15] Paredis J. Co-evolutionary constraint satisfaction.In Parallel Problem Solving from Nature-PPSN III. Springer Berlin Heidelberg, 1994;46-55.

[16] Coello C A C. Use of a self-adaptive penalty approach for engineering optimization problems. Computers in Industry2000;41(2), 113-127.DOI:10.1016/S0166-3615(99)00046-9.

[17] Goldberg DE. (1989). Genetic algorithms in search optimization and machine learning (Vol. 412).Reading Menlo Park: Addison-wesley.

[18] Zhang J, Chung H S H,Lo WL.Clustering-based adaptive crossover and mutation probabilities for genetic algorithms. Evolutionary Computation, IEEE Transactions on2007;11(3), 326-335.

DOI:10.1109/TEVC.2006.880727.

[19] Tessema B, Yen GG. A self adaptive penalty function based algorithm for constrained optimization. In Evolutionary Computation, 2006.CEC 2006. IEEE Congress on (pp. 246-253). IEEE.DOI:10.1109/CEC.2006.1688315.

[20] Nanakor, P, MeesomklinK..An adaptive penalty function in genetic algorithms for structural design optimization. Computers \& Structures2001;79(29), 2527-2539.DOI:10.1016/S0045-7949(01)00137-7. 
[21] Tessema,B, Yen GG. An adaptive penalty formulation for constrained evolutionary optimization.Systems, Man and Cybernetics, Part A: Systems and Humans, IEEE Transactions on2009;39(3), 565-578.

DOI:10.1109/TSMCA.2009.2013333.

[22] Calheiros RN, Ranjan R, Beloglazov A, De Rose CA.,Buyya R. CloudSim: a toolkit for modeling and simulation of cloud computing environments and evaluation of resource provisioning algorithms. Software: Practice and Experience2011;41(1), 23-50.DOI:10.1002/spe.995.

[23] Chen W, Deelman E. Workflowsim: A toolkit for simulating scientific workflows in distributed environments. In E-Science (e-Science), 2012 IEEE 8th International Conference on (pp. 1-8). IEEE. DOI:10.1109/eScience.2012.6404430.

[24] Topcuoglu H, Hariri S, Wu MY. Performance-effective and low-complexity task scheduling for heterogeneous computing. Parallel and Distributed Systems, IEEE Transactions on2002; 13(3), 260-274.

DOI:10.1109/71.993206.

[25] Ostermann,S, Iosup A, Yigitbasi N, Prodan R, Fahringer T, Epema D. A performance analysis of EC2 cloud computing services for scientific computing. In Cloud computing. Springer Berlin Heidelberg,2009;115-131.

[26] Rahman M, Hassan R, RanjanR,Buyya R. Adaptive workflow scheduling for dynamic grid and cloud computing environment. Concurrency and Computation: Practice and Experience2013;25(13), 1816-1842. DOI:10.1002/cpe.3003.

[27] Li Y L, Shao W, Wang J T, et al. An Improved NSGA-II and its Application for Reconfigurable Pixel Antenna Design. Radioengineering, 2014, 23(2):733-738.

[28] Srinivas M, Patnaik L. M. Adaptive probabilities of crossover and mutation in genetic algorithms. IEEE Transactions on Systems, Man, and Cybernetics, 1994;24(4), 656 - 667

[29] Coello C A. Use of a self-adaptive penalty approach for engineering optimization problems. Computers in Industry. 2000; 113-127

[30] Zhu Z, Zhang G, Li M, et al. Evolutionary Multi-Objective Workflow Scheduling in Cloud . IEEE Transactions on Parallel \& Distributed Systems 2016; 27(5):1344-1357.

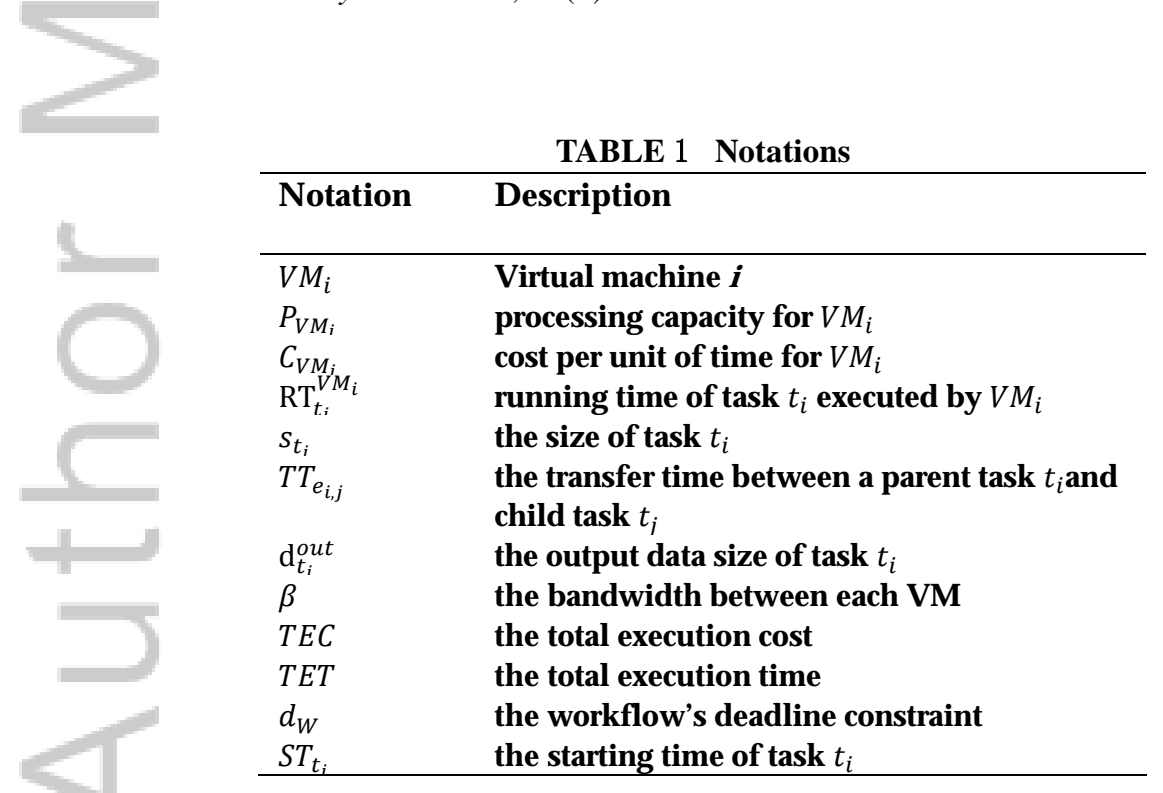




\begin{tabular}{ll}
\hline$E T_{t_{i}}$ & the ending time of task $t_{i}$ \\
$L E T_{V M_{t_{i}}}$ & the lease ending time of $V M_{t_{i^{\prime}}}$ also the time \\
& that $V M_{t_{i}}$ is idle
\end{tabular}

\begin{tabular}{|lccc}
\multicolumn{4}{c}{ TABLE 2. } \\
\cline { 2 - 5 } Name & Types of VMs Used in the Experiments \\
\hline m1.small & 1 & 44 & $\begin{array}{c}\text { Cost per } \\
\text { Hour }\end{array}$ \\
\hline m1.large & 4 & 176 & $\$ 0.03$ \\
m1.xlarge & 8 & 352 & $\$ 0.12$ \\
c1.medium & 5 & 220 & $\$ 0.24$ \\
c1.xlarge & 20 & 880 & $\$ 0.06$ \\
\hline
\end{tabular}

TABLE 3. Performance Comparing of Algorithms under Different Constraints

\begin{tabular}{|c|c|c|c|c|c|c|c|c|c|}
\hline \multirow{2}{*}{\multicolumn{2}{|c|}{ Workflow Algorithm }} & \multicolumn{4}{|c|}{ Deadline 1} & \multicolumn{4}{|c|}{ Deadline 2} \\
\hline & & $\begin{array}{c}\text { Mean } \\
\text { TEC }\end{array}$ & $\begin{array}{c}95 \% \mathrm{CI} \\
\text { TEC }\end{array}$ & $\begin{array}{c}\text { Mean } \\
\text { TET }\end{array}$ & $\begin{array}{l}\text { Meeting } \\
\text { rate }\end{array}$ & $\begin{array}{c}\text { Mean } \\
\text { TEC }\end{array}$ & $\begin{array}{c}95 \% \mathrm{CI} \\
\mathrm{TEC} \\
\end{array}$ & $\begin{array}{c}\text { Mean } \\
\text { TET }\end{array}$ & $\begin{array}{c}\text { Meeting } \\
\text { rate }\end{array}$ \\
\hline \multirow{5}{*}{ Epigenomics } & Random & 100.35 & {$[40.2,160.5]$} & 98475 & $10 \%$ & 100.35 & {$[40.2,160.5]$} & 102099 & $3.3 \%$ \\
\hline & HEFT & 35.12 & {$[35.12,35.1]$} & 13916 & $100 \%$ & 35.10 & {$[35.12,35.12]$} & 13916 & $100 \%$ \\
\hline & GA & 22.73 & {$[12.9,32.5]$} & 20743 & $100 \%$ & 32.98 & {$[12.5,54.5]$} & 25892 & $100 \%$ \\
\hline & PSO & 21.15 & {$[9.2,33.1]$} & 36140 & $100 \%$ & 41.55 & {$[8.1,85.1]$} & 31170 & $100 \%$ \\
\hline & $\mathrm{CGA}^{2}$ & 17.95 & {$[7.2,28.7]$} & 20919 & $100 \%$ & 20.38 & {$[10.7,20.1]$} & 28404 & $100 \%$ \\
\hline \multirow{5}{*}{ Montage } & Random & 52.56 & {$[24.4,80.7]$} & 1233.3 & $90 \%$ & 52.56 & {$[24.38,80.74]$} & 1233.3 & $40 \%$ \\
\hline & HEFT & 18.65 & {$[18.7,18.7]$} & 173.45 & $100 \%$ & 18.65 & {$[18.65,18.65]$} & 173.45 & $100 \%$ \\
\hline & GA & 4.03 & {$[2.45,5.11]$} & 1806.2 & $100 \%$ & 3.98 & {$[2.33,5.63]$} & 1521.0 & $100 \%$ \\
\hline & PSO & 13.7 & {$[6.3,21.1]$} & 1922 & $100 \%$ & 16.21 & {$[10.4,22.0]$} & 1315.6 & $100 \%$ \\
\hline & CGA2 & 3.64 & {$[2.43,4.85]$} & 1362 & $100 \%$ & 4.82 & {$[3.05,6.60]$} & 1082 & $100 \%$ \\
\hline \multirow{5}{*}{ Inspiral } & Random & 52.56 & [6.937.82] & 32762.0 & $90 \%$ & 52.56 & {$[24.38,80.74]$} & 32762 & $40 \%$ \\
\hline & HEFT & 9.83 & {$[9.83,9.83]$} & 4783.56 & $100 \%$ & 9.83 & {$[9.83,9.83]$} & 4783.5 & $100 \%$ \\
\hline & GA & 4.96 & {$[4.64,5.27]$} & 8058.10 & $100 \%$ & 5.33 & {$[5.16,5.50]$} & 8189.0 & $100 \%$ \\
\hline & PSO & 4.24 & {$[4.13,4.35]$} & 12900.0 & $100 \%$ & 4.52 & {$[4.40,4.65]$} & 10610 & $100 \%$ \\
\hline & CGA2 & 4.94 & {$[4.63,5.25]$} & 8678.10 & $100 \%$ & 5.54 & {$[5.38,5.69]$} & 7129.7 & $100 \%$ \\
\hline \multirow{5}{*}{\multicolumn{2}{|c|}{$\begin{array}{cc}\text { Cybershake } & \text { GA } \\
& \text { PSO } \\
& \text { CGA2 } \\
\end{array}$}} & 9.8000 & {$[9.38,10.22]$} & 4130.90 & $13.3 \%$ & 9.8000 & {$[9.38,10.22]$} & 4130.90 & $0 \%$ \\
\hline & & 8.86 & {$[8.86,8.86]$} & 601 & $100 \%$ & 8.86 & {$[8.86,8.86]$} & 601.00 & $100 \%$ \\
\hline & & 4.31 & {$[3.85,4.78]$} & 1776.80 & $100 \%$ & 4.6980 & {$[4.11,5.28]$} & 1567.40 & $100 \%$ \\
\hline & & 3.41 & {$[3.04,3.77]$} & 2133.00 & $100 \%$ & 3.7373 & {$[3.45,4.02]$} & 1858.00 & $100 \%$ \\
\hline & & 4.15 & {$[3.91,4.39]$} & 1767.50 & $100 \%$ & 4.9487 & {$[4.43,5.47]$} & 1483.70 & $100 \%$ \\
\hline \multirow[t]{2}{*}{ Workflow } & Algorithm & \multicolumn{4}{|c|}{ Deadline 3} & \multicolumn{4}{|c|}{ Deadline 4} \\
\hline & & Mean & $95 \% \mathrm{CI}$ & Mean & Meeting & Mean & $95 \% \mathrm{CI}$ & Mean & Meeting \\
\hline
\end{tabular}




\begin{tabular}{|c|c|c|c|c|c|c|c|c|c|}
\hline & & TEC & TEC & TET & rate & TEC & TEC & TET & rate \\
\hline \multirow{4}{*}{ Epigenomics } & Random & 98.37 & {$[40.2,160.5]$} & 98475 & $0 \%$ & 87.43 & {$[40.2,160.5]$} & 110509 & $0 \%$ \\
\hline & HEFT & 35.12 & {$[35.12,35.1]$} & 13916 & $100 \%$ & 35.12 & {$[35.12,35.12]$} & 13916 & $100 \%$ \\
\hline & GA & 46.98 & {$[20.8,73.6]$} & 20743 & $93.3 \%$ & 97.69 & {$[54.2,141.3]$} & 22745 & $73.3 \%$ \\
\hline & PSO & 80.28 & {$[28.5,132.1]$} & 36140 & $26.7 \%$ & 124.16 & {$[65.9,184.4]$} & 76206 & $6.7 \%$ \\
\hline+1 & $\mathrm{CGA}^{2}$ & 23.06 & {$[15.2,30.9]$} & 20919 & $100 \%$ & 25.28 & {$[11.7,38.8]$} & 16807 & $80 \%$ \\
\hline \multirow{5}{*}{ Montage } & Random & 52.56 & {$[24.38,80.7]$} & 1233.3 & $10 \%$ & 52.56 & {$[24.38,80.74]$} & 1233.3 & $0 \%$ \\
\hline & HEFT & 18.65 & {$[18.65,18.7]$} & 173.45 & $100 \%$ & 18.65 & {$[18.65,18.65]$} & 173.45 & $100 \%$ \\
\hline & GA & 13.6 & {$[8.64,18.62]$} & 486.4 & $80 \%$ & 21.65 & [11.63,31.7] & 779.5 & $20 \%$ \\
\hline & PSO & 28.9 & {$[16.8,40.1]$} & 611.6 & $70 \%$ & 41.6 & {$[35.7,46.5]$} & 1085.5 & $10 \%$ \\
\hline & $\mathrm{CGA}^{2}$ & 5.87 & {$[4.05,7.69]$} & 448.9 & $100 \%$ & 18.9 & {$[8.9,30.9]$} & 435 & $60 \%$ \\
\hline \multirow{5}{*}{ Inspiral } & Random & 52.56 & {$[24.4,80.7]$} & 32762.0 & $10 \%$ & 52.56 & {$[24.38,80.74]$} & 32762 & $0 \%$ \\
\hline & HEFT & 9.83 & {$[9.83,9.83]$} & 4783.5 & $100 \%$ & 9.83 & {$[9.83,9.83]$} & 4783 & $100 \%$ \\
\hline & GA & 9.99 & [ 9.25,10.73] & 6233.3 & $93.3 \%$ & 11.457 & {$[10.0,12.9]$} & 6803.7 & $33.3 \%$ \\
\hline & PSO & 6.77 & {$[6.42,7.13]$} & 18650.0 & $73.3 \%$ & 8.1353 & {$[7.02,9.24]$} & 28300 & $16.6 \%$ \\
\hline & CGA2 & 6.46 & {$[6.21,6.71]$} & 6247.6 & $100 \%$ & 6.6580 & {$[6.31,7.00]$} & 5504.5 & $96.7 \%$ \\
\hline \multirow{5}{*}{ Cybershake } & Random & 9.8000 & {$[9.38,10.22]$} & 4130.90 & $0 \%$ & 9.8000 & {$[9.38,10.22]$} & 4130 & $0 \%$ \\
\hline & HEFT & 8.86 & {$[8.86,8.86]$} & 601.00 & $100 \%$ & 8.86 & {$[8.86,8.86]$} & 601.00 & $100 \%$ \\
\hline & GA & 6.4400 & {$[6.19,6.68]$} & 922.96 & $100 \%$ & 10.796 & {$[8.82,12.77]$} & 922.96 & $86.7 \%$ \\
\hline & PSO & 4.8933 & {$[4.56,5.22]$} & 1200.00 & $100 \%$ & 25.001 & {$[22.5,27.47]$} & 1200.0 & $33.3 \%$ \\
\hline & CGA2 & 6.3680 & {$[6.04,6.69]$} & 815.99 & $100 \%$ & 7.4060 & {$[6.84,7.96]$} & 815.99 & $100 \%$ \\
\hline
\end{tabular}




\section{University Library}

\section{- M M I N E R VA A gateway to Melbourne's research publications}

Minerva Access is the Institutional Repository of The University of Melbourne

Author/s:

Liu, L;Zhang, M;Buyya, R;Fan, Q

Title:

Deadline-constrained coevolutionary genetic algorithm for scientific workflow scheduling in cloud computing

Date:

2017-03-10

Citation:

Liu, L., Zhang, M., Buyya, R. \& Fan, Q. (2017). Deadline-constrained coevolutionary genetic algorithm for scientific workflow scheduling in cloud computing. CONCURRENCY AND COMPUTATION-PRACTICE \& EXPERIENCE, 29 (5), https://doi.org/10.1002/cpe.3942.

Persistent Link:

http://hdl.handle.net/11343/291707 